\title{
Commerce et développement de l'élevage laitier en Afrique de l'Ouest : une synthèse
}

\author{
G. Duteurtre *
}

\begin{abstract}
Mots-clés
Bovin laitier - Elevage - Production laitière - Commercialisation Politique de développement - Afrique occidentale.
\end{abstract}

\begin{abstract}
Résumé
Depuis 30 ans, le développement des industries laitières urbaines a bouleversé l'organisation du commerce des produits laitiers en Afrique de l'Ouest. Répondant à une demande urbaine en forte croissance, elles ont suscité la croissance rapide des importations de poudre de lait bon marché. Pourtant, l'élevage laitier local a connu depuis 15 ans d'importantes transformations qui remettent en cause ce modèle industriel " déconnecté » de la production locale. Les mutations de l'élevage laitier ouest africain sont liées à l'apparition, à la fin des années 1990, de minilaiteries artisanales et industrielles tournées vers la collecte de lait local. Pour répondre à ces nouveaux débouchés, les systèmes d'élevage paysans (pasteurs, agropasteurs ou agroéleveurs) se sont engagés dans des systèmes laitiers semi-intensifs basés sur la complémentation alimentaire des vaches laitières. Parallèlement, un nombre croissant d'étables urbaines et de fermes laitières se sont développées à proximité des centres urbains, sur des modèles parfois plus intensifs. Face à la concurrence de la poudre de lait importée, ces nouveaux acteurs des filières laitières se sont positionnés sur des segments de marchés valorisant l'origine locale des produits. La constitution de ces nouveaux circuits de collecte et de transformation entraîne progressivement des évolutions dans les politiques de développement laitier. De nouveaux modes de régulation des échanges apparaissent, encouragés par la mise en place d'instances de concertation entre l'Etat et les acteurs privés. Ces nouvelles options concernent la remise en cause progressive des politiques d'ouverture des marchés, la promotion de modèles techniques d'élevage moins coûteux en intrants, et la mise en place de dispositifs participatifs de gestion de la qualité des produits au sein des filières.
\end{abstract}

\section{- INTRODUCTION : LA FAIBLE CONNEXION DE LA PRODUCTION LAITIERE AFRICAINE AU SECTEUR INDUSTRIEL}

Au cours des vingt dernières années, le secteur laitier a connu de profondes transformations dans les pays du Sud. Alors que la production laitière régressait très sensiblement en Europe, elle a augmenté en moyenne de près de 60 p. 100 en Asie, 45 p. 100 en Afrique et en Océanie, et 40 p. 100 en Amérique du Sud (tableau I). Ce

\footnotetext{
* Rudec, Bat 2G, 298 Kim Ma, Hanoi, Vietnam ; Cirad, UR Systèmes d'élevage, TA C-18 / A, Campus international de Baillarguet, 34398 Montpellier Cedex 5, France.

E-mail : duteurtre@cirad.fr
}

« fleuve blanc » a résulté à la fois d'une augmentation des rendements laitiers et de l'accroissement du troupeau (tableau II).

L'essor de la production laitière dans les pays de l'hémisphère Sud s'est ainsi traduit par un mouvement de concentration de la production dans des régions laitières spécialisées, orientées vers l'approvisionnement d'industries $(14,19)$. A côté de ce grand mouvement d'intensification, d'autres régions d'élevage, au contraire, ont abandonné leur orientation laitière pour s'investir dans de nouvelles activités économiques ou pour participer au grand mouvement d'exode rural $(14,42)$. Parallèlement, des régions laitières plus ou moins isolées ont su conserver un avantage compétitif en dehors du modèle de production de masse, grâce à des produits de qualité spécifique : fromage, beurre, produits frais typiques. Ces filières localisées ont en particulier bénéficié du développement des petites et moyennes agro-industries rurales $(7,54)$. 
L'essor de la production laitière dans les pays en développement s'est accompagné d'une profonde réorganisation des marchés et des filières, induite par des politiques libérales. En vingt ans, les importations laitières dans le monde ont triplé (tableau III). De nombreuses industries urbaines se sont développées sur la base de ce commerce, utilisant de la poudre de lait là où le lait local était insuffisant ou n'était pas disponible, pour approvisionner en produits transformés les villes du Sud en plein essor. De grands groupes, comme Nestlé, Fonterra, Daily Farmers of America, Dean Foods, Danone, ou Lactalis, se sont recomposé. Ces firmes ont notamment investi dans de nouveaux outils industriels localisés en partie dans les pays en développement. Elles ont aussi adopté des stratégies de joint venture et ont pénétré ces nouveaux marchés par l'intermédiaire de licences de marques (14).

L'Afrique subsaharienne n'a pas échappé à cette évolution. La production laitière $\mathrm{y}$ a connu une augmentation rapide, particulièrement en Afrique de l'Est (figure 1). Des pays comme le Kenya ont même développé de manière conséquente leurs exportations de produits laitiers (5). Cependant, en Afrique de l'Ouest, les filières laitières africaines ont été marquées par l'utilisation massive de la poudre de lait importée (figure 2). Dans

\section{Tableau I}

Evolution de la production laitière mondiale depuis 20 ans (millions de tonnes)

\begin{tabular}{lccc} 
& $\begin{array}{c}\text { Production } \\
\mathbf{1 9 8 8}\end{array}$ & $\begin{array}{c}\text { Production } \\
\mathbf{2 0 0 8}\end{array}$ & $\begin{array}{c}\text { Variation } \\
\mathbf{1 9 8 8 - 2 0 0 8} \\
\mathbf{\%}\end{array}$ \\
\hline Afrique & & & $+44,2$ \\
Amériques & 20,3 & 36,4 & $+32,9$ \\
$\quad$ Amérique du Sud & 113,8 & 169,7 & $+38,7$ \\
Asie & 30,2 & 49,3 & $+39,8$ \\
Europe & 99,4 & 247,2 & $+59,2$ \\
Océanie & 281,1 & 215,8 & $-30,2$ \\
Total monde & 14,1 & 24,5 & $+42,4$ \\
& 528,7 & 693,7 & $+23,7$
\end{tabular}

Source : Faostat, 2009

\section{Tableau II}

Evolution du nombre d'animaux laitiers depuis 20 ans (millions de têtes)

\begin{tabular}{lccc} 
& $\begin{array}{c}\text { Nb. d'animaux } \\
\text { laitiers en 1988 }\end{array}$ & $\begin{array}{c}\text { Nb. d'animaux } \\
\text { laitiers en 2008 }\end{array}$ & $\begin{array}{c}\text { Variation } \\
\mathbf{1 9 8 8 - 2 0 0 8} \\
\mathbf{\%}\end{array}$ \\
\hline Afrique & 110,6 & 179,4 & $+38,3$ \\
Amériques & 52,9 & 59,2 & $+10,6$ \\
Amérique du Nord & 11,6 & 10,5 & $-10,5$ \\
Amérique du Sud & 35,6 & 40,4 & $+11,8$ \\
Asie & 232,0 & 362,3 & $+35,9$ \\
Europe & 130,8 & 79,3 & $-64,9$ \\
Océanie & 42,9 & 61,3 & $+30,0$ \\
Total monde & 530,6 & 686,4 & $+22,7$
\end{tabular}

Source : Faostat, 2009 la plupart des pays, les élevages locaux ont connu une transition marchande, caractérisée par des difficultés d'accès aux marchés (27). Par ailleurs, la part des importations dans le disponible laitier a fortement augmenté, représentant aujourd'hui près de 40 p. 100 (tableau IV).

Le secteur laitier en Afrique de l'Ouest a ainsi été marqué par une certaine déconnexion entre la production laitière locale et l'industrie laitière. Cette situation de blocage du développement de la production locale a constitué un des fils conducteurs des initiatives d'appui à l'élevage africain des années 1960 à aujourd'hui. La plupart des opérations de développement laitier se sont focalisées sur les solutions techniques à promouvoir pour " rattraper» ce retard : amélioration génétique, cultures fourragères, rationnement des vaches laitières, promotion de centres de collecte réfrigérés. Il s'agissait alors de susciter l'essor de systèmes de production « intensifs », capables de répondre à l'augmentation rapide de la demande urbaine. Or, faute d'une connaissance approfondie des conditions dans lesquelles devaient être mises en place ces innovations, un grand nombre de ces projets n'ont pas abouti à l'augmentation attendue de la productivité $(45,55,56)$.

Le constat de ces échecs a encouragé le développement de recherches prenant en compte l'environnement technique, économique et social des activités laitières pour mieux comprendre les logiques des systèmes laitiers en place $(10,31)$. Ces nouvelles approches ont bénéficié de réflexions menées plus globalement sur les relations entre commerce et développement $(29,42,55)$.

Qu'en est-il aujourd'hui des débats sur les perspectives d'intégration marchande de l'élevage africain ? Les contraintes à la commercialisation du lait local sont-elles en passe d'être levées ? Des

\section{Tableau III}

Ratio d'évolution des importations laitières en valeur entre 1988 et 2007

$\begin{array}{lc} & \begin{array}{c}\text { Ratio } \\ \text { valeur } \mathbf{2 0 0 7} / \text { valeur } \mathbf{1 9 8 8}\end{array} \\ \text { Afrique } & 2,49 \\ \text { Amériques } & 3,13 \\ \text { Asie } & 3,55 \\ \text { Europe } & 2,60 \\ \text { Océanie } & 4,95 \\ \text { Monde } & 2,82\end{array}$

Source : Faostat, 2009

\section{Tableau IV}

Part de la production locale dans le disponible laitier total (production + importations) en Afrique subsaharienne

\begin{tabular}{lcccccc} 
Année & $\mathbf{1 9 6 1}$ & $\mathbf{1 9 6 7}$ & $\mathbf{1 9 7 7}$ & $\mathbf{1 9 8 7}$ & $\mathbf{1 9 9 7}$ & $\mathbf{2 0 0 7}$ \\
\hline Afrique de l'Est (\%) & 97 & 95 & 94 & 93 & 96 & 97 \\
Afrique centrale (\%) & 82 & 79 & 70 & 63 & 75 & 74 \\
Afrique du Sud (\%) & 100 & 98 & 98 & 92 & 88 & 90 \\
Afrique de l'Ouest (\%) & 87 & 84 & 58 & 61 & 75 & 63 \\
Total (\%) & 96 & 93 & 86 & 86 & 89 & 87
\end{tabular}

Source : Faostat, 2009 


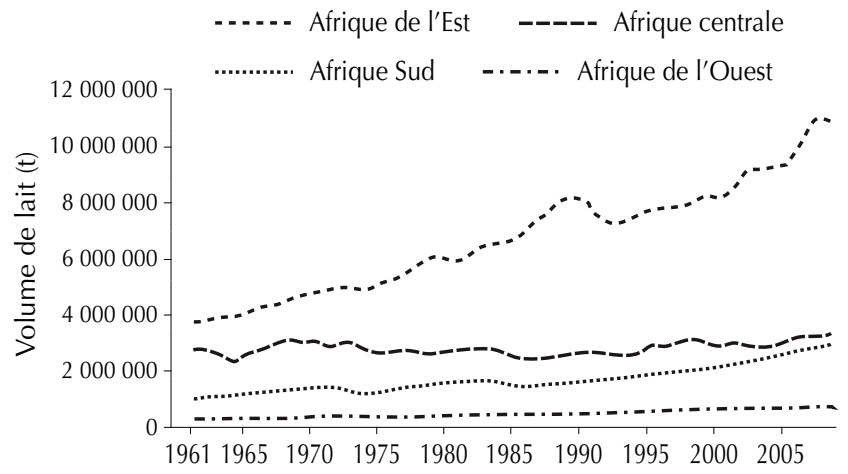

Figure 1 : évolution de la production laitière en Afrique subsaharienne depuis 1961 (en tonnes). Source : Faostat, 2009.

recherches récentes ont permis de faire le point sur l'état actuel de ce secteur emblématique. Plusieurs de ces travaux menés en Afrique de l'Ouest sont publiés dans le présent numéro spécial $(6,8$, $11,12,16,30,33,35,39,40,41,46$ à 51). Trois éléments principaux ressortent de ces recherches : l'émergence récente d'entreprises privées de transformation laitière rurales ; la grande diversité des modèles techniques de production laitière ; et la complexité des arrangements institutionnels qui régissent les relations entre acteurs dans les filières. Ces trois éléments sont présentés dans cet ordre, afin de souligner l'importance des déterminants en aval ( $1^{\text {re }}$ partie) sur l'évolution des élevages ( $2^{\mathrm{e}}$ partie). L'analyse des arrangements institutionnels ( $3^{\mathrm{e}}$ partie) permet de suggérer plusieurs options politiques susceptibles d'encourager l'intégration des systèmes laitiers paysans dans l'industrie laitière locale.

\section{EMERGENCE RECENTE DES INDUSTRIES LAITIERES PRIVEES}

L'émergence récente des entreprises privées de transformation laitière en Afrique s'explique par les réformes politiques successives qui ont touché l'ensemble des économies de la sous-région et par les évolutions de la demande urbaine en produits laitiers. Trois périodes distinctes peuvent être identifiées dans l'histoire laitière africaine : la période coloniale, les décennies postindépendances et la période libérale.

\section{Politiques coloniales et commerce à longue distance}

Sous le régime colonial, les autorités encouragèrent la mise en place d'infrastructures de transport, de points d'eau pastoraux et de marchés qui facilitèrent le développement de petites entreprises de collecte du lait et de transformation. On assista à la mise en place de beurreries tenues par des français, comme celle de Massokory au Tchad ou celle de Fataladji dans le Soudan français, ou même de petites laiteries et fromageries collectant du lait de brousse. Mais ces entreprises privées étaient majoritairement destinées à alimenter le marché des expatriés, encore très étroit, avec des produits de type européen (yaourt, beurre pasteurisé, fromage).

Or, dans les villes et les villages, la consommation de produits laitiers concernait essentiellement des produits de type traditionnel : lait frais, lait fermenté, beurre fermier et huile de beurre. Ces produits étaient fabriqués au sein d'exploitations pastorales ou agropastorales et faisaient l'objet d'échanges locaux importants. Le développement des infrastructures coloniales et des réseaux de commerçants de brousse facilitèrent l'épanouissement de ces échanges. A la faveur de la croissance des villes, un commerce de

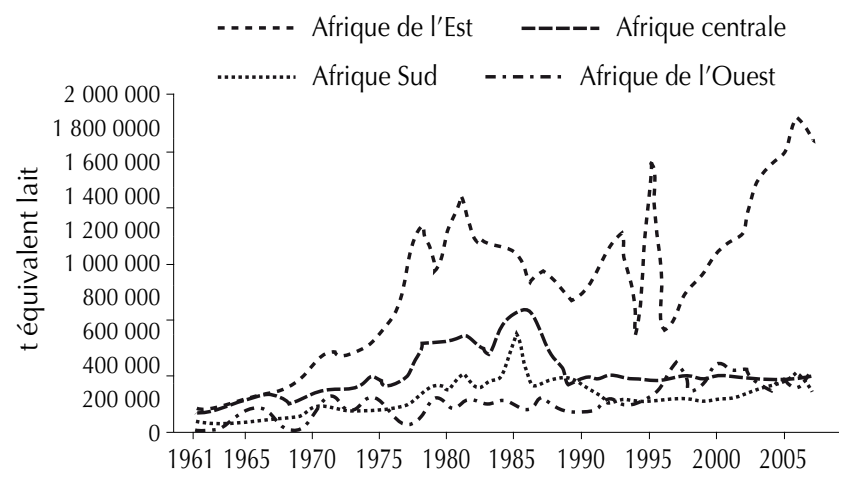

Figure 2 : évolution des importations de produits laitiers en Afrique subsaharienne depuis 1961 (en tonnes équivalent lait). Source : Faostat, 2009.

moyenne distance se mit progressivement en place, orienté depuis les marchés de brousse vers les marchés urbains (55).

Ces échanges prirent aussi la forme de circuits commerciaux à longue distance. Au Tchad, par exemple, plusieurs milliers de tonnes de beurre clarifié étaient acheminées dans les années 1930 à travers le désert vers l'Egypte et le Moyen-Orient. Pendant la Seconde Guerre mondiale, un courant d'exportation du beurre tchadien fut même développé vers la France et l'Angleterre, via les ports du Congo ou du Nigeria. Les exportations d'huile de beurre vers la métropole atteignirent près de 2000 tonnes en 1944. A partir de la fin des années 1960, ces expéditions se tarirent petit à petit. Cet âge d'or du négoce du beurre clarifié au Tchad prit des formes relativement similaires dans des pays comme le Niger, le Mali ou le Sénégal, mais dans des dimensions plus modestes (30).

Ainsi, dès cette époque, les systèmes pastoraux furent confrontés à la logique du marché et au commerce international, mais en tant que fournisseur d'un produit d'export, c'est-à-dire dans des termes opposés à ceux que l'on connaît aujourd'hui. Les politiques menées dans les années 1960-70 et l'évolution du contexte international expliquent en partie le renversement qui suivit.

\section{Politiques postindépendances et capitalisme d'Etat}

Après les Indépendances, les politiques économiques furent marquées par la réorganisation du commerce au travers de sociétés publiques, et par des mesures d'administration des marchés. D'un modèle de capitalisme marchand, on passa à un capitalisme d'Etat. Dans le secteur laitier, de nombreux pays d'Afrique s'engagèrent dans la mise en place de « fermes d'Etats » et de « laiteries gouvernementales ». Très peu d'entreprises privées de collecte ou de transformation furent encouragées à se développer. Ce fut l'époque où furent créés l'Union laitière de Bamako (ULB), l'Union des coopératives laitières (Ucolait) de Saint-Louis, ou encore l'Office laitier du Niger (Olani), qui procédaient de ce modèle industriel impulsé par les pouvoirs publics et les bailleurs de fonds (tableau V).

Ces industries possédaient la plupart du temps le monopole de la collecte du lait frais, dont le prix d'achat était administré. Cependant, les laiteries gouvernementales s'investirent assez faiblement dans l'appui technique aux producteurs des zones rurales, préférant tabler sur l'installation de fermes intensives périurbaines. Les projets de mise en place de centres de collecte se heurtèrent en effet à diverses difficultés de gestion propres aux sociétés étatiques, ainsi qu'à de nombreux obstacles techniques. La collecte pastorale, si elle paraissait envisageable pour de petites entreprises, représentait en fait un véritable défi pour des unités de transformation de grande taille localisées en milieu urbain $(6,24,55)$. 
Cette situation coïncida avec le déversement sur le marché mondial d'importants stocks de poudre de lait. Une partie de ces surplus fut bradée sous forme de dons alimentaire, tandis qu'un courant d'importation commerciale se créait petit à petit dans presque tous les pays de la sous-région. Dans ce nouveau schéma, les unités industrielles utilisèrent de plus en plus de poudre de lait, alors qu'elles avaient été mises en place dans un souci de développer la production locale.

Par ailleurs, la période postindépendance fut marquée au Sahel par les sécheresses de 1973 et 1984-85 qui eurent pour conséquence de réduire très sensiblement la production laitière pastorale. Le « Sahel vert » laissa ainsi la place à un « Sahel en crise » et l'on assista à une baisse de la contribution des systèmes pastoraux à l'économie laitière régionale.

Malgré cela, les circuits informels de commercialisation des produits laitiers pastoraux continuèrent à fonctionner. Il s'agissait en fait des circuits qui existaient de longue date et que les politiques d'administration des marchés n'avaient pas pris en compte : vente de proximité du lait frais, commerce du lait caillé et du beurre sur les marchés hebdomadaires. En réponse à la croissance de la demande urbaine, ces circuits se développèrent autour des grandes villes de la zone soudano-sahélienne (31).

\section{Politiques libérales et émergence des petites entreprises laitières}

Face aux difficultés de fonctionnement des grandes entreprises d'Etat et pour répondre aux recommandations des bailleurs de fonds, les gouvernements s'engagèrent, à partir des années 1980, dans des programmes de libéralisation et de privatisation de leur économie. Dans cette dérégulation des marchés qui suivit les programmes d'ajustement structurel, les grandes laiteries industrielles furent privatisées, les quotas d'importation furent supprimés et le commerce du lait fut libéralisé. Ces nouvelles politiques suscitèrent le développement d'entreprises laitières privées. La compétitivité du lait local fut par ailleurs améliorée par la dévaluation du franc CFA en janvier 1994 (45).

C'est à cette époque que se mit en place en Afrique de l'Ouest et du Centre le modèle des minilaiteries artisanales. Ces minilaiteries se développèrent surtout à partir de la fin des années 1990 dans les villes secondaires du Sénégal, du Mali, du Burkina Faso

\section{Tableau V}

Exemples d'industries laitières d'Etat créées après les Indépendances

\begin{tabular}{llcccc} 
Pays & Industrie & $\begin{array}{c}\text { Date de } \\
\text { création }\end{array}$ & $\begin{array}{c}\text { Capacité de traitement } \\
\text { (L/jour) }\end{array}$ & $\begin{array}{c}\text { Collecte de lait local } \\
\text { (L/jour) }\end{array}$ & $\begin{array}{c}\text { Date de } \\
\text { privatisation }\end{array}$ \\
\hline Mali & ULB (Union laitière de Bamako) & 1969 & 30000 & 5400 (en 1991) & 1994 (Mali-lait) \\
Niger & Olani (Office laitier du Niger) & 1970 & 40000 & 300 (en 1970) & 1998 (Solani) \\
Sénégal & $\begin{array}{l}\text { Ucolait } \\
\text { (Complexe laitier de Saint-Louis) }\end{array}$ & 1968 & 10000 & 550 (en 1971) & Dépôt de bilan \\
& & & & en 1974
\end{tabular}

Source : AlimenTerre, 2008 ; Metzger et coll., 1995 ; Vatin, 1996

Tableau VI

Typologie des minilaiteries et laiteries mises en place dans les années 1990 et 2000

\begin{tabular}{|c|c|c|c|c|c|c|}
\hline $\begin{array}{l}\text { Type } \\
\text { d'entreprise }\end{array}$ & $\begin{array}{l}\text { Zone } \\
\text { d'implantation * }\end{array}$ & $\begin{array}{l}\text { Collecte } \\
\text { (L/jour) }\end{array}$ & $\begin{array}{c}\text { Utilisation } \\
\text { poudre de lait }\end{array}$ & $\begin{array}{l}\text { Electricité } \\
\text { (frigo) }\end{array}$ & $\begin{array}{l}\text { Equipement } \\
\text { industriel }\end{array}$ & $\begin{array}{l}\text { Type de produit } \\
\text { commercialisé }\end{array}$ \\
\hline $\begin{array}{l}\text { Collectrices - } \\
\text { transformatrices }\end{array}$ & Rurale/urbaine & 10 à 60 & Parfois & Non & Non & $\begin{array}{c}\text { Lait frais, lait fermenté, } \\
\text { beurre liquide }\end{array}$ \\
\hline Centre de collecte & Rurale & 50 à 400 & Non & Non & Non & $\begin{array}{c}\text { Lait frais, lait fermenté, } \\
\text { beurre }\end{array}$ \\
\hline $\begin{array}{l}\text { Unité de } \\
\text { pasteurisation } \\
\text { artisanale }\end{array}$ & Rurale/urbaine & 50 à 600 & Parfois & Oui & Non & $\begin{array}{l}\text { Lait frais en sachet, } \\
\text { lait fermenté en sachet, } \\
\text { beurre liquide }\end{array}$ \\
\hline Fromagerie & Rurale & 50 à 300 & Non & Oui & Non & $\begin{array}{l}\text { Fromage frais ou tome } \\
\text { (de vache et de chèvre) }\end{array}$ \\
\hline $\begin{array}{l}\text { Minilaiterie } \\
\text { industrielle }\end{array}$ & $\begin{array}{l}\text { Rurale (ou urbaine } \\
\text { avec collecte rurale) }\end{array}$ & $\begin{array}{c}1000 \text { à } \\
5000 \text { L/jour }\end{array}$ & Parfois & Oui & Oui & $\begin{array}{l}\text { Lait pasteurisé, } \\
\text { lait fermenté, crème } \\
\text { fraîche, jus de fruits }\end{array}$ \\
\hline $\begin{array}{l}\text { Laiterie } \\
\text { industrielle } \\
\text { urbaine }\end{array}$ & Urbaine & $\begin{array}{c}\text { Capacité : } \\
20000 \text { à } \\
60000 \text { L/jour }\end{array}$ & Oui & Oui & Oui & $\begin{array}{l}\text { Yaourt, lait UHT, lait } \\
\text { fermenté, crème } \\
\text { fraîche, jus de fruits }\end{array}$ \\
\hline
\end{tabular}

Source : Broutin et coll., 2007 ; Dieye et coll., 2009 ; Duteurtre, 2009 ; Metzger et coll., 1995

* La zone rurale inclut ici les campagnes et les espaces faiblement urbanisés, y compris les ceintures laitières situées en périphérie des villes secondaires. 
et du Niger. Il s'agissait en fait d'entreprises de formes variées : collectrices - transformatrices, centres de collecte, unités de pasteurisation ou fromageries (tableau VI). Ces ateliers bénéficièrent de l'appui de projets mais surtout de démarches entrepreneuriales. Ils prirent la forme d'entreprises individuelles ou, plus rarement, d'associations, de groupements d'intérêts économiques, ou de coopératives laitières $(22,23,24)$.

Au Sénégal, par exemple, le nombre d'unités de pasteurisation passa de 10 à 40 entre 2000 et 2005. Au Mali, les minilaiteries du réseau Danaya Nono installées dans plusieurs villes secondaires furent parmi les premières à produire du lait pasteurisé ou fermenté en sachet thermosoudé. Elles testèrent plusieurs solutions innovantes pour renforcer les liens entre entreprises de collecte et producteurs laitiers. Plus récemment, la laiterie de Kasséla constitua un exemple intéressant de centre de collecte artisanal organisé sous forme de coopérative, appuyé par un projet. Ce centre développa de manière originale un système de crédit en intrants. Comme pour la plupart des minilaiteries artisanales, les producteurs s'organisèrent entre eux pour l'acheminement du lait grâce à des collecteurs à vélo (49). Ces minilaiteries mobilisèrent de façon plus ou moins importante la poudre de lait en vrac pour compléter les approvisionnements de saison sèche. Les fromageries furent parmi les seules unités laitières à ne travailler qu'à partir du lait local, en raison des difficultés techniques liées à la maîtrise du caillage lactique du lait reconstitué.

On vit aussi émerger un petit nombre de minilaiteries industrielles collectant en zones rurale, comme la Laiterie Tiviski en Mauritanie (en 1989), la Laiterie Faso-Kossam au Burkina Faso (en 1991) ou, plus récemment, la Laiterie du Berger au Sénégal (en 2007). Ces entreprises se différencièrent des unités de pasteurisation par leur capacité de transformation et par leur niveau d'équipement. Elles étaient pourvues de chaines de froid, de pasteurisateurs et de lignes d'ensachage semi-automatiques (tableau VI).

Ces nouvelles entreprises privées contribuèrent à faire émerger en zone rurale un marché du lait de collecte, créant ainsi de nouveaux débouchés pour les éleveurs des zones pastorales et agropastorales. De nombreux projets s'intéressèrent alors à la promotion des ceintures laitières autour des villes secondaires, dans des environnements restant principalement ruraux.

\section{Essor des industries urbaines et du secteur de la distribution}

A l'inverse, le secteur de la grande transformation industrielle resta majoritairement déconnecté de la production laitière locale. Les grandes laiteries d'Etat furent toutes privatisées, avec plus ou moins de réussite, et poursuivirent, pour la plupart, des stratégies de production basées sur l'utilisation de poudre de lait importée. Cette « bataille industrielle » fut en particulier très vive à Dakar : entre 2000 et 2005, le nombre d'industries de reconditionnement de la poudre passa de 1 à 7, et le nombre d'usines laitières fabriquant du yaourt de 1 à 4 (11).

Tandis que la population urbaine était en plein essor (tableaux VII et VIII), le segment des produits de masse fut l'objet d'une compétition industrielle aiguë. Pour répondre à la demande en forte croissance, de nouvelles marques de lait en poudre apparurent, présentées dans des emballages variés : boîtes, cartons, sachets, microsachets. Les industries urbaines élaborant des produits à bas prix à partir de poudre de lait importée rivalisèrent d'ingéniosité pour développer leur gamme de produits et investirent des sommes énormes dans la publicité et le marketing.

La dynamique « d'accroissement et de diversification de l'offre de produits laitiers » s'appuya aussi sur le secteur de la distribution
(11). La poudre de lait bon marché et facile d'utilisation fut la base du développement d'une foison de microentreprises de proximité pour la plupart évoluant dans le secteur informel : revendeuses des marchés, cantines, bars, restaurants. Ces microentreprises urbaines s'adaptèrent rapidement aux nouvelles demandes des villes et commercialisèrent des microsachets de poudre, des boissons lactées, du lait caillé reconstitué ou des bouillies de céréales. Par ailleurs, les boutiques des marchés et les supermarchés des grandes villes se firent les relais de l'élargissement de la gamme des produits industriels en proposant de nombreuses marques de sachets de lait en poudre, de yaourts, de yaourts à boire, de lait pasteurisé UHT, ou de lait caillé aromatisé (16).

\section{Rapports de prix et compétitivité du lait local}

Parfois plus cher que le lait en poudre reconstitué, le lait local resta néanmoins compétitif dans de nombreux circuits en raison de la diversité des attributs de qualité recherchés par les consommateurs (tableau IX). A Bobo-Dioulasso, par exemple, l'analyse du marché du lait et du yaourt montra que l'hygiène et le goût des produits constituaient deux déterminants essentiels dans les choix des consommateurs en plus du prix (3). A Ouagadougou, une étude révéla que la consommation de lait frais pasteurisé était fortement influencée par le niveau d'appréciation du goût par les consommateurs et par le niveau de disponibilité des produits dans les différents quartiers de la capitale, alors que le prix n'apparaissait pas comme un déterminant significatif des achats (47). Cette même étude souligna que le lait frais pasteurisé et le lait UHT étaient des produits de «luxe » consommés par les ménages à hauts revenus, tandis que le lait caillé et le lait concentrés étaient plutôt des produits de grande consommation.

Cette segmentation du marché fut un élément important de la compétitivité du lait local. A la différence de la poudre importée, présente sur les segments de consommation « de masse », les produits laitiers locaux se positionnèrent ainsi plutôt sur des segments

\section{Tableau VII}

Population totale en Afrique de l'Ouest, de l'Est et du centre de 1961 à 2008 (millions d'habitants)

\begin{tabular}{lrrrr} 
& $\mathbf{1 9 6 1}$ & $\mathbf{1 9 8 1}$ & $\mathbf{2 0 0 1}$ & $\mathbf{2 0 0 8}$ \\
\hline Afrique de l'Ouest & 85,6 & 143 & 243,9 & 291,2 \\
Afrique de l'Est & 84,1 & 147,7 & 269,4 & 310,5 \\
Afrique centrale & 32,8 & 55,4 & 100,8 & 122,5
\end{tabular}

Source : Faostat, 2009

\section{Tableau VIII}

Population urbaine en Afrique de l'Ouest, de I'Est et du centre de 1961 à 2008 (\% du total)

\begin{tabular}{lrlll|}
$\begin{array}{l}\text { Population urbaine } \\
\text { (\% de la pop. totale) }\end{array}$ & $\mathbf{1 9 6 1}$ & $\mathbf{1 9 8 1}$ & $\mathbf{2 0 0 1}$ & $\mathbf{2 0 0 8}$ \\
\hline Afrique de l'Ouest & 15,8 & 27,8 & 39,4 & 43,5 \\
Afrique de l'Est & 7,6 & 15 & 21,1 & 23,2 \\
Afrique centrale & 18,3 & 29,3 & 37,7 & 41,8 \\
\hline
\end{tabular}

Source : Faostat, 2009 
« haut de gamme » : lait cru vendu en ville à un prix double du prix du lait reconstitué, lait frais ou fermenté en sachet thermosoudé, crème fraîche ou fromages. Le marché du lait local fut en particulier tiré par le développement des ateliers de fabrication du lait fermenté en sachet thermosoudé (unité de pasteurisation), et par les petites fromageries élaborant du fromage blanc ou du fromage de chèvre.

Cependant, ces petites entreprises laitières des villes secondaires pénétrèrent difficilement le marché des capitales, en raison notamment de l'éloignement des zones de production agropastorales dans lesquelles elles étaient implantées. Certaines se heurtèrent ainsi à la saturation de leurs débouchés locaux $(11,16)$. Seules quelques entreprises laitières rurales parvinrent à proposer un modèle industriel reliant les systèmes pastoraux aux marchés des capitales comme, par exemple, Tiviski en Mauritanie, la Laiterie du Berger au Sénégal, ou Mali-lait au Mali. Mais ce modèle industriel « intégré » s'appuya sur une utilisation mixte du lait local et de la poudre de lait comme matière première, qui prit des formes différentes suivant les entreprises et les lieux de leur implantation (16).

La consommation de lait local fut aussi dopée par la restauration hors foyer : bars laitiers, cantines, restaurants de rue produisant des produits typiques. L'essor des bars laitiers à N'Djamena procéda de cette dynamique d'émergence de filières localisées. Ces boutiques urbaines proposèrent aux consommateurs de la capitale des produits typiques à consommer sur place, à des prix très supérieurs au lait en poudre reconstitué : le halib (lait frais entier) et le rayeb (lait fermenté entier). L'origine pastorale de ces produits constituait un élément essentiel de leur compétitivité (39).

Dans la plupart des villes situées à proximité de zones d'élevage, le lait local montra ainsi une forte résistance vis-à-vis de la concurrence de la poudre de lait sur certains segments de marché très spécifiques. Ces mutations des marchés eurent des répercussions importantes sur l'amont des filières. Les différents systèmes de production laitière impliqués dans l'approvisionnement des villes ne se positionnèrent pas tous de la même manière vis-à-vis de cette nouvelle demande urbaine.

\section{DIVERSITE DES MODELES TECHNIQUES DE PRODUCTION LAITIERE}

En Afrique de l'Ouest, la production laitière n'est pas uniquement destinée au commerce. Le lait est issu en majorité d'exploitations pastorales et agropastorales pour lesquelles la participation à une collecte de lait constitue un cas particulier et non pas le cas général. On estime, par exemple, que seulement 7 p. 100 du lait produit au Sénégal fait l'objet d'une collecte par des unités de transformation (tableau X). La proportion est probablement assez similaire dans les autres pays sahéliens, même si l'on manque de données précises pour l'évaluer.

De fait, alors que les statistiques nationales évaluent la production laitière en terme de collecte dans la plupart des pays développés, elle est estimée en terme de production en Afrique de l'Ouest. La production laitière totale de la sous-région s'élèverait ainsi à environ 2,4 millions de tonnes, pour un cheptel de 50 millions de bovins. Mais la majeure partie de ce lait serait autoconsommée ou échangée localement (38). La question du devenir de l'élevage laitier dans la sous-région se pose ainsi en termes d'augmentation de la production, mais aussi en termes de connexion des systèmes agricoles aux circuits de collecte, c'est-à-dire en termes d'accès aux marchés. Les conditions de vies des ménages agricoles apparaissent ainsi dépendantes à la fois des quantités produites et des opportunités de vente.

Or, pour la majeure partie des exploitations laitières africaines, la production laitière est l'objet d'arbitrages complexes entre les besoins des veaux, l'alimentation de la famille et les échanges économiques. La compréhension de ces arbitrages passe par une analyse fine des systèmes techniques et sociaux dans le cadre desquels est assurée la production laitière.

\section{Le lait dans les systèmes pastoraux et agropastoraux}

Les exploitations pastorales et agropastorales d'Afrique de l'Ouest regroupent une très grande diversité de profils. Le lait provient d'unités de production extrêmement hétérogènes du point de vue du foncier disponible, du nombre d'animaux, de leur potentialité génétique,

\section{Tableau IX}

Prix d'achat du lait local et de quelques autres produits laitiers en 2005 dans différents pays d'Afrique de I'Ouest

\begin{tabular}{lcccc} 
Produit & Prix de vente (Fcfa */L) & Lieu & Année & Source \\
\hline Lait en poudre reconstitué (détail) & 200 à 225 & Dakar (Sénégal) & 2005 & Broutin et coll., 2007 \\
& 250 & Ouagadougou (Burkina) & 2005 & Agéco, 2006 \\
Lait de collecte (rural) & 250 & Kolda (Sénégal) & 2005 & Broutin et coll., 2007 \\
& 200 à 250 & Bobo-Dioulasso (Burkina) & 2005 & Hamadou et coll., 2008 \\
& 200 à 300 & Nord Burkina & 2005 & Agéco, 2006 \\
Lait cru (ferme périurbaine) & 400 & Dakar (Sénégal) & 2005 & Broutin et coll., 2007 \\
& 400 & Ouagadougou (Burkina) & 2005 & Agéco, 2006 \\
Lait cru (détail) & 600 & Dakar (Sénégal) & 2005 & Broutin et coll., 2007 \\
& 400 à 600 & Ouagadougou (Burkina) & 2005 & Agéco, 2006 \\
Lait importé UHT (détail) & 800 & Ouagadougou (Burkina) & 2005 & Agéco, 2006 \\
Lait local fermenté (détail) & 825 & Dakar (Sénégal) & 2005 & Broutin et coll., 2007 \\
& 1000 & N'Djamena (Tchad) & 2005 & Koussou et coll., 2007 \\
& 1250 & Ouagadougou (Burkina) & 2005 & Agéco, 2006
\end{tabular}

$* 1 €=655,957$ Fcfa en 2005 


\section{Tableau X}

Production et collecte de lait au Sénégal en 2007 (en millions de litres)

\begin{tabular}{lccc}
$\begin{array}{l}\text { Type de } \\
\text { production }\end{array}$ & $\begin{array}{c}\text { Production } \\
\mathbf{2 0 0 7}\end{array}$ & $\begin{array}{c}\text { Collecte } \\
\mathbf{2 0 0 7}\end{array}$ & $\begin{array}{c}\text { Part de la collecte } \\
\text { sur le total produit } \\
\text { (\%) }\end{array}$ \\
$\begin{array}{l}\text { Production } \\
\text { extensive ou } \\
\text { semi-intensive } \\
\text { (races locales } \\
\text { en zones rurales) }\end{array}$ & 111,5 & 3,6 & 3,3 \\
$\begin{array}{l}\text { Production } \\
\text { intensive (laitières } \\
\text { exotiques ou métisses } \\
\text { en périurbain) }\end{array}$ & 4,5 & 4,5 & 100 \\
$\begin{array}{l}\text { Total } \\
\text { Tot }\end{array}$ & & \\
\hline
\end{tabular}

Source : Bureau du lait, 2008 (estimations)

* Lait collecté par les laiteries, les minilaiteries ou livré à Dakar. La collecte s'entend ici non compris les échanges sur les marchés ruraux et le commerce de produits dits traditionnels qui représentent néanmoins une part du lait commercialisé.

du système d'alimentation, du niveau de mobilité du troupeau et de la famille, de la part du lait dans les revenus, ou de l'importance des activités agricoles et non-agricoles (18). Autour de Bobo-Dioulasso, par exemple, cette diversité s'illustre par la contribution de pasteurs transhumants, d'agropasteurs sédentaires ou d'agriculteurs, à l'approvisionnement laitier de la ville à partir des terroirs agricoles environnants. Entre 70 et 80 p. 100 d'entre eux considèrent le lait comme une production secondaire sur leur exploitation (36).

Dans la plupart des systèmes pastoraux et agropastoraux, le lait est, avant tout, un produit de l'exploitation utilisé à de nombreuses fins, avant d'être une marchandise. Il est ainsi un élément important de la croissance des jeunes bovins et peut, à ce titre, être volontairement laissé au veau, notamment en périodes de sécheresse. Il constitue par ailleurs une composante essentielle du régime alimentaire des familles qui le consomment sous forme de boisson ou de plats préparés. Il est en outre utilisé pour la confection de lait fermenté, de beurre ou d'huile de beurre, ou même de fromage dans le nord du Niger et du Mali. Ces produits, en grand partie autoconsommés, font l'objet d'échanges divers. Enfin, si le marché le permet, le lait peut aussi être commercialisé sous forme de lait cru $(8,15,55)$.

En outre, le troupeau constitue un capital sur pied dont la gestion dépasse largement son utilisation pour la production de lait. La conduite des animaux s'inscrit dans des systèmes techniques complexes : gestion des pâturages,valorisation des sous-produits agricoles, production de la fumure, besoins en traction, ou forme d'accumulation des revenus agricoles et non agricoles (18). De plus, les animaux constituent un élément fondamental dans les relations sociales entretenues au sein et en dehors de la famille. Ils constituent des objets de don, de confiage ou peuvent être vendus pour faire face aux dépenses de la famille. Les éleveurs sont donc amenés à arbitrer entre plusieurs objectifs d'utilisation du troupeau : alimentation de la famille, revenus monétaires, projets d'équipements, maintien et transmission du patrimoine, participation à la vie de la communauté. En milieu pastoral et agropastoral, les animaux et leurs productions ont donc des objectifs multifonctionnels (28).

Dans les environnements africains, la mise en marché du lait doit être envisagée dans un contexte de forte contrainte sur les systèmes de production : la gestion collective du troupeau, la saisonnalité des ressources fourragères agropastorales, la mobilité des animaux, et les difficultés d'accès aux aliments concentrés constituent autant d'éléments qui rendent difficile le pilotage de l'alimentation des animaux laitiers $(8,18,50)$. Par ailleurs, la fragmentation des droits sur le lait au sein des familles, le faible développement des réseaux de collecte et des moyens de transport, et la diversification des activités économiques au sein des ménages compliquent la mise en place de véritables stratégies de production laitière spécialisée orientées vers la collecte (15). Ainsi, en milieu agropastoral, la valorisation marchande du lait se fait le plus souvent par le biais des ventes locales de produits transformés par les femmes. Ces ventes sont saisonnières et alimentent des circuits orientés vers les marchés urbains $(7,46)$.

Dans ce contexte, la mise en place d'étables laitières et l'élevage d'animaux laitiers croisés avec des races laitières importées constituent des innovations particulièrement complexes à réaliser (50). De fait, le lait provient en majorité d'animaux rustiques adaptés aux conditions agroécologiques locales et aux différentes fonctions du bétail. On rencontre de nombreuses races de zébus dans les zones les plus sèches (Gobra, Peul, Goudali, Azaouak, Djelli, Bororo), des taurins dans les savanes plus humides (N'dama, Baoulé), et de manière plus diffuse des animaux issus de croisements entre zébus et taurins (comme le Djakoré) ou d'autres croisements $(8,9,36)$.

Ces vaches produisent entre un et quatre litres de lait par jour. Cependant, en raison du faible coût des ressources et de la main d'œuvre, les coûts de production de tels systèmes sont assez bas. Ils ont par exemple été évalués à $150 \mathrm{Fcfa} / \mathrm{L}$ en moyenne en milieu agropastoral au Sénégal (50). Cette production reste saisonnière et dispersée, et les coûts de collecte sont donc relativement élevés.

Un certain nombre de systèmes de production laitière évoluent néanmoins vers des systèmes plus engagés dans le marché, mais ces changements sont progressifs. Ils prennent des formes très différenciées en fonction des ressources fourragères disponibles, des potentialités génétiques des animaux, des savoir-faire des éleveurs, ou des débouchés disponibles. Dans bien des cas, les systèmes laitiers paysans s'appuient sur des techniques d'élevage semi-intensif.

\section{Elevage paysan et systèmes laitiers semi-intensifs}

En zones pastorales et agropastorales, les pratiques d'élevage évoluent surtout en périphérie des villes et autour des minilaiteries rurales, c'est-à-dire là où le lait fait l'objet d'un commerce régulier et sécurisé. Le développement des ceintures laitières périurbaines repose ainsi en grande partie sur la présence de collecteurs à vélo, de détaillantes - transformatrices, et d'unités de transformation. Certains de ces acteurs en aval sont parfois eux-mêmes issus de familles d'agroéleveurs et possèdent ainsi une bonne connaissance de l'amont. Ces intermédiaires jouent ainsi un rôle de mise en relation entre les nouvelles demandes urbaines et la production agropastorale $(16,46,49)$.

Les systèmes paysans semi-intensifiés reposent essentiellement sur l'utilisation de compléments alimentaires disponibles localement pour augmenter la production de lait. On observe notamment dans plusieurs zones le recours croissant aux graines de coton, aux tourteaux de coton, aux fanes d'arachide, aux sons de céréales, aux pailles et aux autres résidus de récoltes $(8,18)$. L'utilisation de compléments va de pair avec la pratique d'allotement des animaux laitiers (parfois qualifiée de stabulation laitière) qui peuvent être alors séparés du reste du troupeau pendant les périodes de transhumance $(15,18,26)$.

En périphérie de Ségou, par exemple, l'allotement d'un nombre plus important de vaches laitières et l'utilisation de concentrés alimentaires permettent à certains agroéleveurs peuls ou bambara 
de commercialiser des quantités importantes de lait. Cet engagement laitier participe d'évolutions très lentes de l'organisation familiale (parfois sur plusieurs générations). Les fonctions des revenus laitiers évoluent elles aussi : les nouvelles entrées monétaires sont mobilisées par les hommes pour les dépenses familiales, mais aussi pour l'entretien du troupeau. Les femelles laitières sont exploitées soit en gestion directe au sein de la famille, soit par l'intermédiaire de bergers salariés. La transhumance apparaît parfois comme une réponse à la saturation des terroirs et à la capitalisation du bétail. On observe alors des pratiques de délocalisation du troupeau laitier en périphérie des ville. La « marchandisation » de ces systèmes suppose ainsi des modifications importantes dans l'organisation des familles $(15,18,46)$.

En revanche, ces évolutions impliquent des changements techniques relativement faibles au sein des unités de production : " soutien de la production en saison sèche par des concentrés achetés, distribution de fourrages stockés, de pailles de céréales (résidus de culture) ou de foin de brousse » (46). Certains éleveurs utilisent parfois des animaux de race métisse (croisement avec des zébus Maures, notamment), mais de manière marginale. La plus grosse partie du lait reste produite par des animaux de race locale. Les changements de pratiques sont ainsi peu novateurs, « ni par rapport aux évolutions des techniques de production du lait chez les Peuls plus ou moins sédentarisés d'Afrique de l'Ouest, ni par rapport à l'histoire de l'élevage laitier dans le monde » (46). Ils sont essentiellement mis en œuvre en réponse à une péjoration des conditions d'alimentation au pâturage (suite à une forte occupation des sols pour l'agriculture et à des charges animales élevées) et à la demande du marché. Ces évolutions de pratiques laitières résultent finalement plus de l'adaptation locale des techniques d'embouche que d'une réplique d'un modèle laitier intensif (46).

De ce fait, les systèmes de complémentation animale adoptés par les éleveurs sont très spécifiques à chaque zone. Ainsi, en région agropastorale du Niger, le sel et le son de céréales constituent les compléments alimentaires de base des troupeaux laitiers. Mais les autres compléments utilisés diffèrent d'une zone à l'autre. Dans les environs de Niamey, les agropasteurs utilisent prioritairement de la paille de brousse, du foin de bourgou, du natron, et des résidus de cultures sur champ, tandis que dans la zone de Filingué, située à $175 \mathrm{~km}$ de Niamey, les agropasteurs utilisent plutôt de la graine de coton et du tourteau de coton (8).

Certains auteurs parlent d'élevages à faibles intrants (EFI) pour caractériser les unités de production laitière agropastorales faiblement intensifiées, pratiquant notamment la complémentation des vaches laitières. A Bobo-Dioulasso, ces systèmes contribuent pour 98,3 p. 100 à l'approvisionnement en lait de la capitale régionale (36). Ils se différencient des élevages à visée commerciale (EVC) qui présentent des niveaux d'intensification beaucoup plus élevés. Même si ces EVC conduisent leurs animaux au pâturage, ils constituent en fait des unités très différentes des exploitations familiales agropastorales. Plutôt que d'EVC, nous les qualifierons d'exploitations laitières urbaines et périurbaines. On peut toutefois souligner que, dans le cas des élevages localisés autour de Bobo-Dioulasso, Hamadou et coll. (36) reconnaissent que la part du lait commercialisé au sein des EFI (78 à 83 p. 100 de la production totale) est très proche de la part commercialisée par les EVC (79 à 100 p. 100 de la production). Ces deux types d'exploitations sont donc en fait à visée commerciale et leur différenciation se fait sur des critères plus complexes.

\section{Exploitations laitières urbaines et périurbaines}

Autour des capitales et à l'intérieur des quartiers urbains on assiste au développement d'élevages laitiers d'un type nouveau. Il s'agit essentiellement d'étables urbaines ou de fermes laitières. Les étables urbaines sont des unités de production généralement sommaires, installées de manière précaire dans les interstices du tissu urbain ; les fermes laitières sont des unités beaucoup plus intensifiés, orientées vers la production de lait frais. Entre ces deux types d'élevages laitiers, on trouve un continuum d'exploitations plus ou moins intensifiées et plus ou moins bien insérées dans leur environnement. Par ailleurs, dans les zones d'extension urbaine récente, ces nouveaux élevages avoisinent parfois des unités agropastorales plus traditionnelles $(8,18,35)$.

Les étables urbaines ont été décrites dans la plupart des capitales ouest africaines $(45,56)$. Elles souffrent souvent du manque d'espace et exploitent les rares pâturages disponibles sur les espaces publics. Elles sont parfois amenées à fonctionner en élevage horssol (zero grazing), lorsque des aliments fourragers et concentrés sont disponibles localement. En Afrique de l'Ouest, ces étables urbaines sont essentiellement constituées d'animaux de races locales. Elles sont conduites soit par des « nouveaux éleveurs » urbains pouvant faire appel à l'occasion à des bergers pour la conduite du troupeau, soit par des pasteurs ou agropasteurs récemment installés en zones urbaines $(6,8)$.

Les fermes laitières urbaines et périurbaines présentent des profils souvent très différents de ceux des exploitations agropastorales. Elles se caractérisent par une maîtrise des techniques de production modernes ou intensives. Ces producteurs utilisent en particulier, partiellement ou exclusivement, des vaches de races importées (Holstein, Montbéliardes, Brunes des Alpes, etc.) ou métisses, nécessitant l'utilisation de nombreux intrants alimentaires et vétérinaires. Ces éleveurs s'appuient sur des niveaux d'investissement beaucoup plus importants que ceux mobilisés par les exploitations agropastorales. En plus de l'élevage laitier, ces exploitations pratiquent aussi parfois l'embouche bovine, l'élevage avicole, ou l'horticulture. Il s'agit souvent d'investisseurs d'origine urbaine que l'on qualifie aussi de nouveaux éleveurs : fonctionnaires, vétérinaires privés, commerçants, retraités $(8,18,36)$. En périphérie de Bamako, par exemple, où l'on compte de nombreux néo-éleveurs, 42 p. 100 des producteurs de la zone auraient eu recours en 2006 à l'amélioration génétique de leur cheptel. Ces pratiques d'amélioration ont d'ailleurs reposé quasi exclusivement sur des croisements par monte naturelle avec des animaux métis, et non pas sur le recours à l'insémination artificielle $(6,18)$.

Parmi ces fermes laitières, celles disposant de ressources foncières et de main d'œuvre suffisantes mettent parfois en œuvre des cultures fourragères annuelles ou pérennes. Mais ces cultures sont le fait d'éleveurs jeunes, organisés, ou ayant bénéficié d'appuis de la part de projets de développement (34). Par ailleurs, les fourrages cultivés restent relativement peu développés dans toute la sous-région (52).

$\mathrm{Au}$ bout du compte, les exploitations laitières périurbaines présentent de bonnes performances techniques, mais leurs performances économiques sont très hétérogènes. Autour de Bobo-Dioulasso et de Dakar, par exemple, les coûts de production de ces fermes laitières modernes sont une à trois fois plus élevés que les coûts de production des fermes agropastorales. Ces faibles performances financières tendent à remettre en cause leur durabilité et leur capacité à se développer sur le long terme, dans un environnement soumis à une forte concurrence sur le foncier $(6,23,35)$ (tableau XI).

\section{Elevage laitier, diversification des revenus et accès aux marchés}

L'élevage est une activité ancienne en Afrique de l'Ouest qui connaît actuellement de profondes évolutions. Les systèmes d'élevage agropastoraux contribuent de manière croissante à l'approvisionnement des marchés urbains, grâce à la mise en place de 
systèmes d'élevage semi-intensifs. La pression sur les ressources pastorales et l'attraction du marché semblent constituer les deux principaux facteurs d'évolution de ces exploitations familiales. Ces transformations permettent une diversification des revenus des paysans, mais elles génèrent aussi d'importantes réorganisations au sein des familles $(15,18,46)$.

Les systèmes laitiers paysans semi-intensifs constituent ainsi des modèles d'évolution alternatifs par rapport aux systèmes techniques proposés par la plupart des projets d'appui à l'élevage. Les gouvernements et les agences de développement ont, la plupart du temps, promu des systèmes laitiers intensifs, basés sur des programmes d'amélioration génétique, sur la mise en place de cultures fourragères et sur la spécialisation laitière des exploitations. Dans certaines zones périurbaines, des exploitations intensives se mettent en place en s'inspirant de ce modèle moderniste, du fait de l'engagement de nouveaux investisseurs dans des activités d'élevage laitier. Mais ces systèmes laitiers modernes apparaissent encore fragiles en raison de coûts de production élevés et d'une pression croissante sur le foncier périurbain. Ils sont donc souvent

\section{Tableau XI}

Caractéristiques des différents types de systèmes de production laitière en Afrique de l'Ouest (en 2004 et 2005)

\begin{tabular}{|c|c|c|c|c|}
\hline & $\begin{array}{l}\text { Pastoral et } \\
\text { agropastoral }\end{array}$ & $\begin{array}{l}\text { Semi-intensif } \\
\text { paysan }\end{array}$ & $\begin{array}{c}\text { Etable } \\
\text { urbaine }\end{array}$ & $\begin{array}{l}\text { Ferme } \\
\text { laitière }\end{array}$ \\
\hline $\begin{array}{l}\text { Race } \\
\text { locale }\end{array}$ & +++ & +++ & +++ & +++ \\
\hline $\begin{array}{l}\text { Race } \\
\text { métisse }\end{array}$ & - & - & + & +++ \\
\hline \multicolumn{5}{|c|}{ Coût de production } \\
\hline $\begin{array}{l}\text { Centre } \\
\text { Sénégal }\end{array}$ & $\begin{array}{c}150 \\
\text { Fcfa */L }\end{array}$ & $\begin{array}{c}136-170 \\
\text { Fcfa/L }\end{array}$ & & $\begin{array}{c}136 \\
\text { Fcfa/L }\end{array}$ \\
\hline $\begin{array}{l}\text { Nord } \\
\text { Sénégal }\end{array}$ & $\begin{array}{c}144 \\
\text { Fcfa/L }\end{array}$ & & $\begin{array}{c}270 \\
\text { Fcfa/L }\end{array}$ & \\
\hline $\begin{array}{l}\text { Burkina } \\
\text { Faso }\end{array}$ & & & & $\begin{array}{c}100-450 \\
\text { Fcfa/L }\end{array}$ \\
\hline $\begin{array}{l}\text { Spécialisation } \\
\text { laitière }\end{array}$ & Non & Non & Oui & Non \\
\hline
\end{tabular}

Source : Ba Diao et coll., 2006 ; Hamadou et coll., 2008 ; Sow et coll., 2007 $* 1 €=655,957$ Fcfa en 2005 le fait d'éleveurs pluriactifs. Finalement, on constate qu'en Afrique de l'Ouest, le modèle laitier intensif peine à s'imposer au sein de l'agriculture familiale, à la différence des évolutions décrites en Afrique de l'Est, dans le Maghreb, en Amérique latine ou en Asie $(5,32,48,51)$.

La question de l'avenir de l'élevage laitier marchand en Afrique de l'Ouest est donc relativement complexe. Elle ne saurait se réduire à un blocage de l'intensification laitière. De nouveaux travaux sont nécessaires pour mieux comprendre les déterminants internes de la monétarisation des systèmes, en analysant plus finement les logiques productives et leurs bases socio-économiques. Par ailleurs, la prise en compte des déterminants externes de l'accès aux marchés nécessite de s'intéresser de manière transversale aux filières et aux territoires dans lesquels s'insèrent la production laitière (48). La grande hétérogénéité des situations de production s'explique en particulier par la complexité des arrangements entre acteurs au sein des filières laitières.

\section{COMPLEXITE DES ARRANGEMENTS ENTRE ACTEURS AU SEIN DES FILIERES}

Le lait est un produit très périssable qui justifie le recours à de nombreux arrangements entre acteurs pour réduire les risques commerciaux et satisfaire la demande des consommateurs $(14,55)$. Une grande diversité d'institutions, d'organisations, et de référentiels de qualité structurent les filières et sous-filières laitières. Elles impliquent en retour des mécanismes complexes de gestion publique de ce secteur.

\section{Diversité des chaines de collecte et de transformation}

On trouve en Afrique de l'Ouest quatre types de circuits de commercialisation du lait : les ventes de proximité de lait, le négoce de produits fermiers ou traditionnels, les circuits des détaillantes - transformatrices, et les réseaux de collecte des laiteries (tableau XII).

Ces différents circuits constituent des modes alternatifs de commercialisation du lait. Ils peuvent être mobilisés par les éleveurs de manière exclusive ou simultanée. Ils opèrent donc à la fois en concurrence et en complémentarité les uns des autres. Les trajectoires de construction de bassins laitiers industriels montrent souvent un passage progressif de l'un à l'autre de ces modes de commercialisation : vente directe, puis collecte artisanale, puis collecte industrielle (48). Mais même dans les zones fortement industrialisées, on constate que les différents modes alternatifs de commercialisation du lait continuent à coexister $(3,11)$. Au Pérou, par exemple, les circuits industriels ne collectent que la moitié de la production laitière nationale. L'autre moitié de la production est

Tableau XII

Caractéristiques des différents types de circuits de commercialisation du lait

\begin{tabular}{|c|c|c|c|c|}
\hline & Vente de proximité & $\begin{array}{c}\text { Négoce de produits } \\
\text { fermiers ou traditionnels }\end{array}$ & $\begin{array}{c}\text { Détaillante - } \\
\text { transformatrice }\end{array}$ & $\begin{array}{l}\text { Collecte } \\
\text { laiterie }\end{array}$ \\
\hline Stabilité des prix d'achat du lait & +++ & - & + & +++ \\
\hline Niveau du prix d'achat du lait cru & +++ & + & + & ++ \\
\hline Stabilité saisonnière du débouché & + & + & ++ & +++ \\
\hline Importance des contrats liés & ++ & - & ++ & +++ \\
\hline Capacité à collecter dans des zones enclavées & - & +++ & + & ++ \\
\hline Capacité à commercialiser de grandes quantités & + & ++ & + & +++ \\
\hline
\end{tabular}


commercialisée soit sous forme de vente directe de lait cru, soit par l'intermédiaire des négociants de fromages fermiers ou des petites fromageries (3).

Les ventes de proximité de lait frais et de lait caillé se développent majoritairement en ville et constituent le principal débouché pour les étables urbaines et les fermes périurbaines $(8,36)$. Le lait fais ou caillé peut faire l'objet de ventes directes du producteur au consommateur. Mais ces produits sont aussi transportés et livrés au porte-à-porte par des vendeuses ambulantes, qui sont parfois des femmes d'éleveurs (16). Dans certaines villes, les vendeuses de proximité commercialisent aussi leurs produits sur les marchés urbains ou au bord des grands axes de circulation. Par ailleurs, la crème ou le beurre sont fréquemment commercialisés à côté du lait caillé. De même, certaines revendeuses proposent aussi du couscous ou des bouillies (16). Les ventes sur les marchés permettent aussi d'écouler le lait refusé par les laiteries : bidons trop acides ou quantités de lait dépassant les prévisions de transformation (49). Le commerce de proximité du lait et du lait caillé constitue une activité très dynamique dont l'importance est souvent sous-estimée en raison des ventes directes (11).

Le négoce de produits laitiers dits fermiers ou traditionnels constitue un circuit particulièrement dynamique en zone sahélienne. Il repose sur la confection au sein des élevages de lait caillé écrémé, de beurre, d'huile de beurre, ou de fromages (tableau XIII). Ces produits, en raison de leur capacité à se conserver, peuvent être transportés sur de longues distances. Ce négoce participe donc d'une mise en relation des marchés urbains et des zones rurales assez éloignées. Le commerce du lait caillé au Sahel peut ainsi s'étendre sur plusieurs dizaines de kilomètres, grâce à des réseaux de collectrices utilisant des moyens de transport en commun. Le lait caillé est alors généralement transporté dans des bidons en plastique (39). Le commerce de l'huile de beurre au Sahel participe aussi de cette dynamique, mais sur des distances encore plus longues, tout comme le négoce du fromage tchoukou au Niger et au Mali. Ces produits sont susceptibles de faire l'objet d'un commerce transfrontalier $(8,30)$.

Les circuits des détaillantes - transformatrices regroupent des réseaux de collecte très hétérogènes basés sur des techniques de transformation artisanales. Ces transformatrices commercialisent du lait frais, du lait caillé, de la crème ou du beurre. Une des rares études menées sur ces opératrices a concerné la ville de Kasséla, en périphérie de Bamako (49). Trois de ces détaillantes y sont installées et ont développé des réseaux de collecte rurale et de dis- des relations personnelles avec des bergers et alimentent un réseau étroit de revendeuses des marchés et de vendeuses à domicile. Ces petites unités familiales mettent en œuvre des techniques de transformation relativement complexes qui leur permettent de commercialiser un grand nombre de produits : lait chauffé écrémé, lait fermenté écrémé, lait fermenté concentré, crème, huile de beurre. Certaines de ces transformatrices utilisent aussi du lait en poudre dans leur approvisionnement. On trouve aussi dans les capitales africaines un nombre important de transformatrices urbaines ou de cantines qui commercialisent du lait fermenté élaboré exclusivement à partir de poudre de lait (11).

Parmi les circuits de collecte des laiteries, il faut d'abord évoquer le cas des minilaiteries artisanales dont on a vu qu'elles s'étaient fortement développées depuis 20 ans. Malgré leur faible capacité de collecte, elles ont un impact important sur la production en raison de leur nombre important (17). La plupart des unités reçoivent le lait au quai et les livraisons se font à vélo. Elles établissent avec les producteurs laitiers des contrats de collecte qui leur permettent de sécuriser leurs approvisionnements. Elles fournissent aussi souvent des intrants à crédit à ces producteurs afin de limiter les variations saisonnières de l'offre. Les systèmes de collecte mis en place par les minilaiteries industrielles sont assez similaires, mais le transport se fait en général en camionnette.

Les réseaux de collecte des laiteries industrielles urbaines constituent le dernier type de circuits orientés vers les laiteries. La plupart de ces laiteries ne collectent du lait local qu'en quantité réduite par rapport au lait reconstitué à partir de poudre de lait. Or, deux éléments permettent d'entrevoir l'essor d'une collecte plus importante de lait local par ces usines : l'intérêt croissant de certains industriels pour le lait local, motivés par la recherche d'une meilleure image et d'une meilleure qualité pour leurs produits, et l'installation récente d'une minilaiterie industrielle en zone pastorale du Sénégal (la Laiterie du Berger) qui semble constituer un modèle durable, malgré les difficultés techniques d'un tel projet (16).

Dans ces nouvelles configurations, le lait local et la poudre de lait apparaissent parfois plus complémentaires qu'antagonistes. Pour ces industries, en effet, « la difficulté à collecter du lait de brousse (...) se traduit par un coût élevé de la collecte. En outre, les volumes demeurent bien en deçà de leurs capacités de production. Pour rentabiliser une chaîne coûteuse de pasteurisation, elles sont amenées à développer une gamme de jus de fruits (...) voire à transformer du lait en poudre » (16). Le modèle de collecte industrielle du lait local apparaît ainsi comme essentiel dans la constitution de bassins laitiers éloignés des villes, mais il semble aussi favoriser

\section{Tableau XIII}

Nomenclature des produits laitiers dans quelques langues locales d'Afrique de I'Ouest

\begin{tabular}{|c|c|c|c|c|c|c|}
\hline Pays (langue) & $\begin{array}{l}\text { Lait frais issu } \\
\text { de la traite }\end{array}$ & $\begin{array}{c}\text { Lait fermenté } \\
\text { entier }\end{array}$ & $\begin{array}{l}\text { Lait fermenté } \\
\text { écrémé }\end{array}$ & $\begin{array}{l}\text { Lait fermenté } \\
\text { concentré / } \\
\text { crème fraîche }\end{array}$ & $\begin{array}{l}\text { Huile de } \\
\text { beurre }\end{array}$ & Fromage \\
\hline Burkina Faso (Peul) & Biraddam & Kossam & & Tchettu & Nebbam Naï & \\
\hline Mali (Bamabara) & Nono & & & Féné & Sirimé & Tchoukou \\
\hline Mali (Tamachek) & & & & & & Tchikomart \\
\hline Sénégal (Wolof) & Meew & M'bannick & Katch & & Diwu nior & \\
\hline Sénégal (Peul) & & & Kossam Kaadam & Ketoungol & Nebbam Naï & \\
\hline Tchad (Arabe tchadien) & Laban & Rayeb & Rouaba & & Dihin Baggar & \\
\hline Tchad (Peul-fulata) & Kossam & Nialloundé & Pendidam & & Nebbam Naï & \\
\hline
\end{tabular}


l'utilisation du lait en poudre (16) (tableau XII). Pour comprendre ces questions centrales pour l'avenir du secteur laitier en Afrique de l'Ouest, il convient d'analyser plus en détail les relations entre producteurs et transformateurs laitiers.

\section{Contrats de collecte au coeur de la constitution de nouveaux bassins laitiers}

La plupart des livraisons de lait aux unités de transformation se font par l'intermédiaire de contrats de collecte. En effet, la collecte ne peut se résumer à l'achat et au transport du produit. Elle s'accompagne d'une sécurisation de la transaction par différents arrangements institutionnels : accords ou conventions sur les prix et sur la qualité, contrats incluant des avances de trésorerie ou d'intrants, réseaux personnels garantissant la confiance (23). Ces mécanismes de coordination, qui permettent de pallier les incertitudes sur les transactions, sont extrêmement fréquents dans les relations entre producteurs laitiers, collecteurs et entreprises de transformation $(16,48,49)$.

Les contrats de collecte entre les éleveurs et les minilaiteries génèrent d'importants changements dans les systèmes de production. Ils permettent en effet de sécuriser les débouchés laitiers et, ainsi, donnent la possibilité aux éleveurs de mettre en œuvre des innovations permettant de tirer pleinement partie des opportunités marchandes. L'accès aux intrants alimentaires entraîne, notamment, une augmentation du rendement laitier par vache. L'utilisation de graines de coton, de tourteaux, ou d'aliments concentrés permet de complémenter les animaux en lactation. Les avances de trésorerie et la mensualisation du paiement du lait couvrent de nouveaux types de dépenses qui permettent notamment d'investir dans de nouveaux animaux (23).

La mise en place de ces contrats passe aussi par l'émergence de collecteurs laitiers qui se déplacent la plupart du temps à vélo jusqu'aux minilaiteries. Certains bassins laitiers sont organisés autour d'autres moyens de transport (la mobylette autour de N'Djamena, la camionnette pour les petites laiteries industrielles), mais ils impliquent toujours la participation de collecteurs spécialisés assurant l'apport d'une certaine quantité de lait dans un lieu. Ces collecteurs jouent un rôle d'intermédiaires pour le transport du lait, mais aussi de lien social, de colporteurs pour l'achat de biens de consommation, et de garants dans la relation entre industriels et producteurs (20)

Les dynamiques de constitutions de nouveaux bassins laitiers ne sauraient pourtant se réduire à l'efficacité des relations producteurs - collecteurs - transformateurs. De nouvelles recherches sont nécessaires pour mieux comprendre l'insertion de ces nouvelles filières dans les territoires périurbains et ruraux. Il convient en particulier de mieux identifier les facteurs de blocage de l'émergence des bassins de production plus intensifiés, alimentant des unités industrielles : niveau de développement des réseaux de transport, dynamiques locales, sociales et politiques, stratégies d'expansion industrielle, dimension des marchés visés, etc. (48). La grande diversité des circuits de commercialisation du lait et des techniques utilisées rend par ailleurs difficile l'évaluation de la qualité des différents produits commercialisés.

\section{Diversité des référentiels de qualité des produits laitiers}

Le lait est d'abord un produit fragile, susceptible d'être altéré par de nombreuses réactions chimiques et microbiologiques, et de causer des toxi-infections alimentaires collectives. La plupart des recherches sur la qualité des produits laitiers en Afrique de l'Ouest ont, de ce fait, consisté à évaluer l'importance du risque sanitaire lié à l'ingestion des produits laitiers locaux, en essayant de quantifier les taux de germes pathogènes présents dans les produits prélevés à la ferme ou sur les points de vente. Si la plupart des auteurs s'entendent pour reconnaitre le niveau élevé des charges bactériennes des produits laitiers commercialisés tant dans le secteur formel que dans le secteur informel, ils ne s'accordent cependant pas sur les moyens à mettre en œuvre pour régler ce problème $(6,12,24,33,40)$.

La plupart des politiques conduites jusqu'ici en Afrique de l'Ouest ont des démarches coercitives, s'appuyant sur des réglementations contraignantes destinées à protéger le consommateur. Cependant, en Afrique, la mise en place de ce type de politique a butté sur la faiblesse des services de contrôle et de répression. Dans ce contexte, plusieurs projets de développement ont suggéré de promouvoir des démarches « d'incitation à l'autocontrôle de la qualité par les entreprises ou les organisations professionnelles elles-mêmes » (12). Ces programmes ont encore été qualifiés de politiques «d'évaluation participative » de la qualité avec les acteurs concernés $(6,33)$

L'objectif de telles démarches est de proposer des améliorations des pratiques de production, de transformation et de commercialisation du lait par l'élaboration de diagnostics collectifs, suivis de l'identification de bonnes pratiques à promouvoir chez les professionnels et de campagnes d'information. La participation des consommateurs, des transformateurs et des producteurs de lait à de telles démarches collectives, ainsi que la présence active de la recherche et des pouvoirs publics impliqués permettent de déboucher sur des solutions réalistes. Elles pourraient conduire aussi à une meilleure maîtrise des risques pour la santé publique attachés à la consommation des produits laitiers (6).

Au Sénégal et au Burkina Faso, des guides de bonnes pratiques d'hygiène laitière ont ainsi été élaborés avec la participation d'un cercle très large de professionnels et de représentants publics. Ces guides permettent d'appuyer des démarches individuelles d'amélioration de la qualité des produits et peuvent servir de base à des campagnes de formation et d'information des professionnels (12). Au Kenya, l'implication du Smallholder Dairy Project en faveur des petits éleveurs a permis de contrer des campagnes de dénigrement de la qualité des produits laitiers non industriels auprès des consommateurs (33). L'implication des professionnels et des chercheurs a permis de convaincre les pouvoirs publics du bien-fondé de politiques d'encouragement à l'amélioration de la qualité, au détriment des tendances répressives.

Ces démarches participatives d'amélioration de la qualité des produits permettent aussi de limiter les risques d'exclusion sociale de certains acteurs des filières laitières. Les femmes travaillant dans le secteur informel, en particulier, apparaissent très vulnérables face à des politiques coercitives. La promotion de politiques en faveur des pauvres semble ainsi compatible avec de telles démarches basées sur la formation, la sensibilisation et la promotion de bonnes pratiques $(33,49)$.

Dans ce cadre, il apparaît que les normes de qualité à mettre en œuvre sont de nature bien différente des normes habituellement en vigueur dans les pays développés. Parallèlement, la reconnaissance de la typicité des produits fermiers (beurre solide, beurre clarifié, lait caillé, Tchoukou, etc.) soulève la question de l'émergence de réglementations locales propres à défendre la spécificité des produits de terroir (24) (tableau XIII).

Enfin, la diversité des référentiels de qualité explique en partie pourquoi les programmes de promotion du système lactoperoxydase ont rencontré tant d'obstacles en milieu africain, alors que cette innovation apparaissait si prometteuse (41). 
La très grande complexité des filières laitières, tant sur le plant technique que du point de vue socio-économique, implique la recherche d'arbitrages difficiles de la part des services de l'Etat. Cette importance des arbitrages s'illustre notamment dans la gestion des politiques commerciales. Les décisions sur les niveaux de taxation, si elles résultent de l'engagement des pays africains dans les négociations commerciales internationales, apparaissent aussi comme des compromis entre pouvoirs publics et acteurs des filières.

\section{Politiques commerciales comme objet de débats entre Etat et professionnels}

Le lait est emblématique du débat soulevé par l'impact du commerce international sur la pauvreté $(3,51)$. Dans les pays en développement, le secteur élevage est en effet soumis à une ouverture croissante aux importations mondiales (53). Alors que les tarifs douaniers à l'importation des produits laitiers avaient été parmi les plus élevés du monde pendant la période de l'Uruguay Round (1986-94), ils ont été fortement réduits dans le cadre de l'Organisation mondiale du commerce pour atteindre aujourd'hui 25 p. 100 en moyenne (25).

Cette internationalisation des échanges porte en elle des risques de marginalisation grandissante des populations rurales, confrontées à des difficultés d'accès aux marchés $(42,43)$. Les politiques de régulation des marchés laitiers font en particulier l'objet d'intenses débats, aussi bien dans les pays du Nord que ceux du Sud (2). Ces débats ont été d'autant plus vifs au cours des deux dernières années que les prix internationaux du lait ont par deux fois atteint des sommets (37). Produit à forte valence culturelle, sociale et économique, le lait reflète ainsi la fragilité des économies locales face aux nouvelles règles du commerce international (38).

Ces débats ne concernent pas seulement le niveau des barrières tarifaires à promouvoir, mais ils concernent plus largement les modèles d'organisation des filières laitières et la place de l'élevage familial dans ces modèles d'organisation (3). En effet, les choix de politiques commerciales sont indissociables des orientations choisies pour l'ensemble du secteur concerné. Les modèles de développement techniques et organisationnels doivent correspondre à des projets sociaux et économiques qui nécessitent eux-mêmes des configurations tarifaires ou fiscales particulières $(21,25,44)$. Ainsi, dans le cadre du nouveau Tarif extérieur commun, la taxation de la poudre de lait en Afrique de l'Ouest n'aurait pas de sens, sans la remise à plat du modèle laitier industriel qui s'est développé dans la zone et qui inclut le recours massif aux importations pour nourrir les villes (tableau XIV).

\section{Difficiles arbitrages des politiques laitières}

Plutôt qu'organisée autour d'un ou de plusieurs marchés autorégulés, l'économie laitière apparaît comme un emboitement de différents marchés dont les règles de fonctionnement sont le fruit de mécanismes complexes de construction technique et sociale. Ainsi, les solutions politiques les plus pertinentes semblent émerger des processus participatifs, plutôt que des démarches top down. Cela concerne en particulier trois domaines de l'action publique : les projets d'appui technique à la production et aux filières, les politiques d'amélioration de la qualité des produits, et l'ouverture des économies au commerce international. Devant la complexité des solutions, ce qui apparaît comme essentiel pour l'avenir du secteur agricole ouest africain, c'est la mise en place d'instances de concertation entre acteurs des filières (29). La promotion d'organisations professionnelles d'éleveurs et de transformateurs, et la mise sur pied d'organisations interprofessionnelles ou d'instances de concertation constituent ainsi une étape essentielle à l'émergence de nouveaux projets collectifs pour les filières laitières ouest africaines.

Dans certains pays, de telles organisations interprofessionnelles ont permis d'avancer sur les différents sujets de débat. Des tables filières au Burkina Faso, des comités interprofessionnels au Sénégal, ou d'autres types d'interprofessions laitières ont émergé et offrent ainsi de réelles raisons d'espérer (29). Les Journées mondiales du lait, organisées chaque année le premier juin dans plusieurs pays depuis cinq ans, constituent aussi des instances de concertation utiles.

Cependant, des travaux de recherche et d'expertise sont nécessaires pour éclairer ces débats. Il convient en particulier d'évaluer les impacts des recompositions en cours sur les niveaux de pauvreté des différents acteurs et sur les devenir des territoires ruraux. Ces travaux pourront se baser sur une analyse des revenus des acteurs, sur l'élaboration de modèles sectoriels ou sur l'analyse fine des dynamiques territoriales $(3,21,48)$.

\section{Tableau XIV}

Catégories de droits de douane prévues par le TEC

\section{Catégorie de produits}

TEC Uemoa

(en vigueur depuis 2000) (\%)
TEC Cedeao (en discussion en 2009) (\%)

\section{Biens sociaux essentiels}

2. Matières premières de base, produits de première nécessité, biens d'équipement

3. Intrants et produits intermédiaires

4. Biens de consommation finale

5. Produits «vulnérables»

Source : Duteurtre, 2009

TEC : Tarif extérieur commun

Uemoa : Union économique et monétaire ouest africaine (Bénin, Burkina Faso, Côte d'Ivoire, Guinée Bissau, Mali, Niger, Sénégal, Togo)

Cedeao : Communauté économique des Etats d'Afrique de l'Ouest (pays de l'Uemoa + Cap Vert, Gambie, Ghana, Guinée, Liberia, Nigeria, Sierra Leone) 
- CONCLUSION :

\section{ENJEUX D'UNE MEILLEURE CONNEXION} ENTRE ELEVEURS ET INDUSTRIELS

Depuis une vingtaine d'années, l'émergence de nouvelles entreprises de collecte laitière et le développement de la demande urbaine ont impulsé des changements importants au sein des filières laitières. Les systèmes de production laitière paysans bénéficiant d'un débouché sécurisé ont évolué vers des systèmes laitiers semi-intensifs, tandis qu'un petit nombre de fermes urbaines mettaient en œuvre des unités de production plus modernes. Les circuits de commercialisation sont restés relativement diversifiés et la poudre de lait importée a poursuivi son développement, sans remettre en cause cependant les perspectives marchandes du lait local.

Les travaux récents menés sur les filières laitières africaines permettent aujourd'hui de mieux comprendre ces évolutions. Ils soulignent l'urgence de promouvoir une meilleure connexion de la production locale aux unités de transformation industrielles ou semi-industrielles, seules capables de relier les pôles urbains et les bassins d'élevage éloignés. Pour cela, les politiques doivent se positionner à la fois sur les systèmes techniques à promouvoir, sur la place à accorder aux importations et sur les moyens d'améliorer la qualité des produits locaux.

La période récente a semblé marquée par un retour de l'action de l'Etat dans plusieurs domaines de l'espace marchand dans des configurations plutôt favorables à une connexion de la production locale aux industries. D'abord, les discussions nationales et sous-régionales autour du niveau de taxation des importations de produits laitiers ont évolué dans un sens plutôt favorable à un rehaussement des taxes à l'importation du lait liquide (25). Ensuite, l'implication de l'Etat et des agences de développement dans la définition de bonnes pratiques laitières ont permis de renforcer les relations entre éleveurs, collecteurs, unités de transformation et consommateurs. Enfin, la reconnaissance dans certains pays des organisations interprofessionnelles, comme interface entre l'Etat et les acteurs privés des filières laitières et comme mode d'élaboration des priorités politiques, ont offert des perspectives de renouvellement du débat sur les politiques laitières domestiques à mettre en œuvre.

Bien sûr, les arbitrages publics restent encore incertains sur de nombreux sujets relatifs à la régulation des marchés laitiers. Les éleveurs agropastoraux sont restés très prudents vis-à-vis du modèle laitier intensif proposé par de nombreux projets dont on voit qu'il se développe dans de nombreux pays du Maghreb, d'Amérique latine ou même d'Asie. Ce modèle intensif paysan, basé sur l'élevage d'animaux métis et les cultures fourragères, constitue ainsi un des points du débat sur l'avenir de l'élevage laitier en Afrique de l'Ouest. Jusqu'à présent, les systèmes semi-intensifs ont montré le plus de vitalité. De même, les relations entre éleveurs et industriels sont restées encore timides. L'élaboration de nouveaux référentiels de qualité ou la promotion de régimes fiscaux orientés vers la promotion de ces relations constituent, à n'en pas douter, un thème sur lequel les pouvoirs publics et les acteurs des filières devront travailler de concert.

\section{BIBLIOGRAPHIE}

1. AGECO, 2006. Analyse de la problématique de l'importation de la poudre de lait au Burkina Faso et de son effet sur le développement de la filière lait. Rapport de mission, juin 2006. Sainte-Foy, Québec, Ageco, 64 p.

2. ALIMENTERRE, 2008. Pour des politiques européennes cohérentes en faveur des agricultures familiales du Sud. Compte-rendu Séminaire AlimenTerre, Bruxelles, Belgique, 2-8 oct. 2008. Paris, France, AlimenTerre. http://alimenterre08.sosfaim.lu/\#rub_6

3. AUBRON C., 2007. Lait et fromage dans un pays andin : quelle place pour les filières artisanales péruviennes face aux industries laitières? Revue Elev. Méd. vét. trop., 60 : 189-197.

4. BA DIAO M., FALL A.A., SALL C., DIAW O.T., 2006. Influence de la complémentation alimentaire et du déparasitage interne sur le développement économique de la production laitière des vaches Gobra en zone sahélienne du Sénégal. Tropicultura, 24 : 51-57.

5. BERTHELOT J., 2009. Kenya et Niger : la vache qui riz et celle qui pleure. Toulouse, France, Solidarités, $17 \mathrm{p}$.

6. BONFOH B., FOKOU G., OULD TALEB M., FANE A., WOIRIN D. LAIMAIBAO N., ZINSSTAG J., 2007. Dynamiques des systèmes de production, risques et transformations socio-économiques au Mali. Revue Elev. Méd. vét. trop., 60 : 67-76.

7. BOUCHER F., CARIMENTRAND A., REQUIER-DESIARDINS D., 2009. Agro-industries rurales et lutte contre la pauvreté au Pérou. Les systèmes agroalimentaires localisés contribuent-ils au renforcement des " capabilités »? In : Duteurtre G., Faye B., éds, Elevage, richesse des pauvres. Versailles, France, Quae, p. 221-238.

8. BOUKARY A.R., CHAIBOU M., MARICHATOU H., VIAS G., 2007. Caractérisation des systèmes de production laitière et analyse des stratégies de valorisation du lait en milieu rural et périurbain au Niger : cas de la communauté urbaine de Niamey et de la commune rurale de Filingué. Revue Elev. Méd. vét. trop., 60 : 113-120.
9. BOUTRAIS J., 2007. The Fulani and cattle breeds: crossbreeding and heritage strategies. Afr. J. int. Afr. Inst., 77: 18-36.

10. BROKKEN R.F., SENAIT SEYOUM Ed., 1992. Dairy marketing in subSaharan Africa. In: Proc. Symp., ILCA, Addis Ababa, 26-30 Nov. 1990. Addis-Ababa, Ethiopia, ILCA, 392 p.

11. BROUTIN C., DUTEURTRE V., TANDIA A., TOURE B., FRANCOIS M., 2007. Accroissement et diversification de l'offre de produits laitiers au Sénégal : la bataille industrielle du lait en poudre à Dakar et des minilaiteries à la conquête des marchés des villes secondaires. Revue Elev. Méd. vét. trop., $60: 11-19$.

12. BROUTIN B., FRANÇOIS M., LA NOE NICULESCU N., 2007. Gestion de la qualité dans la transformation laitière : expérimentation d'une démarche d'élaboration concertée de guides de bonnes pratiques d'hygiène au Sénégal et au Burkina Faso. Revue Elev. Méd. vét. trop., 60 163-169.

13. BUREAU DU LAIT, 2008. Note sur le secteur laitier au Sénégal. Dakar, Sénégal, Isra-Bame, 5 p.

14. CALVEZ E., 2006. L'économie laitière en France et dans le monde : approche géographique. Rennes, France, Presses universitaires de Rennes, 188 p.

15. CORNIAUX C., 2008. Organisation sociale et zootechnique de la gestion des produits laitiers en milieu sahélien : la sphère laitière. Cas du delta du fleuve Sénégal. Revue Elev. Méd. vét. trop., 61 : 37-43.

16. CORNIAUX C., BONFOH B., DIALLO A., POCCARD-CHAPUIS R., VIAS G., 2007. Réseaux de collecte et de distribution du lait dans les villes d'Afrique soudano-sahélienne. Revue Elev. Méd. vét. trop., 60 21-28.

17. CORNIAUX C., DUTEURTRE G., DIEYE P.N., POCCARD-CHAPUIS R., 2005. Les minilaiteries comme modèle d'organisation des filières laitières en Afrique de l'Ouest : succès et limites. Revue Elev. Méd. vét. trop., 58 : 237-243 
18. COULIBALY D., MOULIN C.H., POCCARD-CHAPPUIS R., MORIN G., SIDIBE S.I., CORNIAUX C., 2007. Evolution des stratégies d'alimentation des élevages bovins dans le bassin d'approvisionnement en lait de la ville de Sikasso au Mali. Revue Elev. Méd. vét. trop., 60 103-111.

19. DELGADO C., ROSEGRANT M., STEINFELD H., EHUI S. COURBOIS C., 1999. Livestock to 2020. The next food revolution. Washington, DC, USA, IFPRI, 17 p.

20. DIA D., DUTEURTRE G., DIEYE P.N., 2007. Le rôle de l'élevage dans la lutte contre la pauvreté : I'exemple des filières laitières locales au Sénégal. In : Mbaye A., Roland-Holst D., Otte J., éds, Agriculture, élevage et pauvreté en Afrique de l'Ouest. Dakar, Sénégal, Crea, p. 103-118.

21. DIARRA A, 2009. Echanges internationaux et développement du secteur laitier sénégalais. Thèse Doct. Economie Université Paris-Sud 11. Montpellier, France, Cirad, 306 p.

22. DIEYE PN., BROUTIN C, DUTEURTRE G, DIA D, 2009. Produits laitiers : importations, industries urbaines et dynamiques artisanales locales. In : Duteurtre G., Faye M.D., Dieye P.N., Coord., L'Agriculture sénégalaise à l'épreuve du marché. Paris, France, Karthala, p. 305-328.

23. DIEYE P.N., MONTAIGNE E., DUTEURTRE G., BOUTONNET J.P. 2008. Le rôle des arrangements contractuels dans le développement du système laitier local et des minilaiteries au Sénégal. Econ. rurale (303304-305) : 108-122.

24. DUTEURTRE G., 2004. Normes exogènes et tradition locale : la problématique de la qualité dans les filières laitières africaines. Cah Agric., 13 ( ${ }^{\circ}$ spécial) : 91-98

25. DUTEURTRE G., 2009. Lait des pauvres, lait des riches : réflexion sur l'inégalité des règles du commerce international. In : Duteurtre G. Faye B., éds, Elevage, richesse des pauvres. Versailles, France, Quae, p. $249-266$

26. DUTEURTRE G., ATTEYEH A., 2000. Le lait à Moundou, témoin de I'intégration marchande des élevages pastoraux au sud du Tchad. Revue Elev. Méd. vét. trop., 53 : 299-306.

27. DUTEURTRE G., BLEIN R., 2009. Afrique de l'Ouest : la révolution de l'élevage aura-t-elle lieu? Grain de Sel (46-47) : 12-14.

28. DUTEURTRE G., FAYE B., coord., 2009. L'élevage, richesse des pauvres : stratégies d'éleveurs et organisations sociales face aux risques dans les pays du Sud. Versailles, France, Quae, 286 p.

29. DUTEURTRE G., FAYE M.D., DIEYE P.N., dir., 2009. L'agriculture sénégalaise à l'épreuve du marché. Paris, France, Karthala, 451 p.

30. DUTEURTRE G., KOUSSOU M., 2007. Economie pastorale et marchés laitiers au Sahel : l'âge d'or du commerce de beurre clarifié au Tchad de 1930 à 1970. Revue Elev. Méd. vét. trop., 60 : 29-38.

31. DUTEURTRE G., MEYER C., éds, 2001. In : Actes atelier Marchés urbains et développement laitier en Afrique subsaharienne, Montpellier France, 10-12 sept. 1998. Montpellier, France, Cirad, 263 p. (Coll. Colloques)

32. GALETTO A., LOPEZ W., BAUMEISTER E., 2007. Competitiveness of milk production in Nicaragua: An analysis of productivity and costs in dual-purpose livestock systems in the Matagalpa Region. Revue Elev. Méd. vét. trop., 60 : 133-140.

33. GRACE D., RANDOLPH T., OMORE A., SCHELLING E., BONFOH B., 2007. Place of food safety in evolving pro-poor dairy policy in Eas and West Africa. Revue Elev. Méd. vét. trop., 60 : 153-162.

34. HAMADOU S., KAMUANGA M., ABDOULAYE T., LOWENBERG DEBOER J., 2005. Facteurs affectant l'adoption des cultures fourragères dans les élevages laitiers périurbains de Bobo-Dioulasso au Burkina Faso. Tropicultura, 23 : 29-35.

35. HAMADOU S., PALE E., DITALAMANE H., 2007. Déterminants de la consommation des produits laitiers à Bobo-Dioulasso (Burkina Faso) facteurs sociaux et sensibilité aux prix. Revue Elev. Méd. vét. trop., 60 51-58.

36. HAMADOU S., TOU Z., TOE P., 2008. Le lait, produit de diversification en zone périurbaine à Bobo-Dioulasso (Burkina Faso) Cah. Agric., $17:$ 473-478

37. IFAD, 2009. Rural poverty report: tentative outline. http://www.ifad org/rural/rpr2010/outline.pdf (consulté le 12.12.2009).
38. ISRA, 2009. Mon lait, je l'aime local : produits et savoir-faire laitiers en Afrique de l'Ouest. Livret de l'exposition itinérante 2009. Dakar, Sénégal, Isra-Bame, $26 \mathrm{p}$.

39. KOUSSOU M.O., DUTEURTRE G., MOPATE L.Y., 2007. Consommation de lait dans les bars laitiers de la ville de N'Djamena au Tchad. Revue Elev. Méd. vét. trop., 60 : 39-44.

40. KOUSSOU M.O., GRIMAUD P., MOPATE L.Y., 2007. Evaluation de la qualité physico-chimique et hygiénique du lait de brousse et des produits laitiers locaux commercialisés dans les bars laitier de N'Djamena au Tchad. Revue Elev. Méd. vét. trop., 60 : 45-49.

41. LHOSTE F., 2007. Limites de l'application de la méthode de conservation du lait cru par le système lactoperoxydase en Afrique de I'Ouest. Revue Elev. Méd. vét. trop., 60 : 171-176.

42. LOSCH B., 2008. La recherche d'une croissance agricole inclusive au cœur de la transition économique africaine. In : Deveze J.C. éd., Défis agricoles africains. Paris, France, Karthala, p. 47-72.

43. MARKELOVA H., MEINZEN-DICK R., HELLIN J., DOHRN S., 2009. Collective action for smallholder market access. Food Policy, 34: 1-7.

44. MERCIER-GOUIN D., 2004. La gestion de l'offre dans le secteur laitier, un mode de régulation toujours pertinent. Sainte-Foy, Québec, Université Laval, Grepa, 134 p.

45. METZGER R., CENTRES J.M., THOMAS L., LAMBERT J.C., 1995. L'approvisionnement des villes africaines en produits laitiers, un potentiel pour le développement rural. Rome, Italie, 102 p. (Production et santé animales 124)

46. MORIN G., COULIBALY D., CORNIAUX C., POCCARD-CHAPPUIS R., SIDIBE S.I., MOULIN C.H., 2007. Dynamiques des unités de production laitière dans le bassin d'approvisionnement de la ville de Ségou au Mali. Revue Elev. Méd. vét. trop., 60 : 89-101.

47. OUEDRAOGO S., DOUANIO H., 2007. Analyse des facteurs qui déterminent la consommation du lait frais local pasteurisé local dans la ville de Ouagadougou au Burkina Faso. Revue Elev. Méd. vét. trop., 60 : 59-65.

48. POCCARD-CHAPUIS R., CORNIAUX C., COULIBALY D., 2007. Dynamiques de structuration des bassins laitiers. Comparaison entre I'Amazonie brésilienne et le Mali. Revue Elev. Méd. vét. trop., 60 : 141 152

49. SCHNEIDER M., KOUYATE H., FOKOU G., ZINSSTAG J., TRAORE A., AMADOU M., BONFOH B., 2007. Dynamiques d'adaptation des femmes face aux transformations des systèmes laitiers périurbains en Afrique de I'Ouest. Revue Elev. Méd. vét. trop., 60 : 121-131.

50. SOW DIA F., SOMDA J., KAMUANGA M., 2007. Dynamique des filières laitières en zone sahélienne : cas de l'offre et de la demande du lait en zone agropastorale centre du Sénégal. Revue Elev. Méd. vét. trop., $60: 77-88$

51. SRAIRI M.T., CHOHIN KUPER A., 2007. Conséquences de la libéralisation des marchés sur les opérateurs de la filière laitière au Maroc. Revue Elev. Méd. vét. trop., 60 : 177-187.

52. TOUTAIN B., KLEIN H.D., LHOSTE P., DUTEURTRE G., 2009. Histoire et avenir des cultures fourragères en Afrique tropicale. Fourrages (200) : 511-523.

53. UPTON M., OTTE J., 2004. The impact of trade agreement on livestock producers. In: Owen E., Smith T., Steele M.A., Anderson S., Duncan A.J., Herrero M., Lever J.D., Reynolds C.K., Richards J.I., Ku-Vera J.C., Eds, Responding to the livestock revolution: the role of globalization and implications for poverty alleviation. Nottingham, UK, Nottingham University Press, p. 51-66.

54. VAN DE KOP P., SAUTIER D., GERZ A., Eds, 2006. Origin-based products: Lessons for pro-poor market development. Bull. Royal trop. Inst. (372): $104 \mathrm{p}$

55. VATIN F., 1996. Le Lait ou la raison marchande, essai de sociologie économique. Rennes, France, Presses universitaires de Rennes, 205 p.

56. WALSHE M.J., GRINDLE J., NELL A., BACHMANN M., 1991. Dairy development in sub-Saharan Africa: A study of issues and options. Washington, DC, USA, The World Bank, 94 p. (Technical paper No 135)

Accepté le 15.03.2010 


\section{Summary}

Duteurtre G. Trade and Development of Dairy Production in West Africa: a Review

In the last 30 years, the development of urban dairy industries has deeply changed the organization of dairy marketing in West Africa. To respond to the high increase of the urban demand, the dairy plants contributed to a fast increase in cheap dry milk imports. However, the domestic dairy production sector has been going through major changes in the past fifteen years. These changes question the industrial model, which is disconnected from the local production. Dairy production changes in West Africa are related to the development, in the late 1990s, of small-scale dairy processing units engaged in the collection of local milk. To respond to this new market outlet, smallholders (pastoral herders, agropastoral farmers) tend to convert to semi-intensive systems, based on feed supplementation of dairy cows. At the same time, a growing number of urban cowsheds and milk farms have developed near urban centers, often with more intensified production systems. To face the competition of imported dry milk, the new actors of the dairy subsector focus on specific market segments that promote the local origin of their products. These new collecting and processing chains are progressively leading to changes in dairy development policies. New trade regulations are emerging between State institutions and private actors. These regulations relate to questioning trade liberalization, to promoting low-input milk production systems, and to setting up participative quality management systems in the dairy marketing chains.

Keywords: Dairy cattle - Animal husbandry - Milk production - Marketing - Development policy - West Africa.

\section{Resumen}

Duteurtre G. Comercio y desarrollo de las crías lecheras en África del Oeste: diagnóstico y opciones políticas

Desde hace 30 años, el desarrollo de las industrias lecheras urbanas ha revolucionado la organización del comercio de los productos lecheros en África del Oeste. Respondiendo a una demanda urbana bajo fuerte crecimiento, han suscitado el rápido crecimiento de las importaciones de leche en polvo barata. Sin embargo, la cría lechera local ha conocido desde hace 15 años importantes transformaciones que ponen en duda este modelo industrial "desconectado" de la producción local. Los cambios de la cría lechera oeste africana están ligados a la aparición, hacia fines de los años 1990, de mini lecherías artesanales e industriales orientadas hacia la colecta de leche local. Para responder a estas nuevas oportunidades, los sistemas de cría campesinos (pastores, agro pastores o agro criadores) se comprometieron con sistemas lecheros semi intensivos, basados sobre la suplementación alimenticia de las vacas lecheras. Paralelamente, un número creciente de establos urbanos y de fincas lecheras se han desarrollado a proximidad de los centros urbanos, siguiendo modelos a veces más intensivos. Frente a la competencia de la leche en polvo importada, estos nuevos participantes de las filiales lecheras, se han localizado en sectores del mercado en que se valora el origen local de los productos. La constitución de estos nuevos circuitos de colecta y de transformación lleva progresivamente a la evolución de las políticas de desarrollo lechero. Nuevos modos de regulación de los intercambios aparecen, impulsados por la aplicación de medios de concertación entre el estado y los participantes privados. Estas nuevas opciones conciernen la puesta en causa progresiva de las políticas de apertura de los mercados, la promoción de modelos técnicos de cría menos onerosos en insumos y la puesta en obra de dispositivos participantes en la gestión de la calidad de los productos en el seno de las filiales.

Palabras clave: Ganado de leche - Ganadería - Producción lechera - Mercadeo - Política de desarrollo - Africa occidental. 